\title{
Team-Centered Virtual Interactive Presence for Adjustable Autonomy
}

\author{
J. Reitsema* and W. Chun ${ }^{\dagger}$ \\ Lockheed Martin Space Systems Co, Denver, CO, 80127 \\ T. Fong \\ NASA Ames Research Center, Mountain View, CA 94035 \\ R. Stiles ${ }^{\S}$ \\ Lockheed Martin Space Systems Co, Sunnyvale, CA, 94089
}

\begin{abstract}
Current solutions for autonomy plan creation, monitoring and modification are complex, resulting in loss of flexibility and safety. The size of ground control operations and the number of aviation accidents involving automation are clear indicators of this problem. The solution is for humans to naturally collaborate with autonomous systems. Visual spatial information is a common reference for increased team situation awareness between humans and adaptive autonomous systems such as robots, satellites, and agents. We use a teamcentered, augmented reality spatial dialog approach to improve human-automation interaction for collaborative assembly, repair, and exploration. With our spatial dialog approach, we provide a data-rich virtual interactive presence for collaborating with autonomous systems by incorporating the spatial context along with the spoken context. Spatial Dialog is an interaction and communication technology that uses a deeper understanding of spatial context and a richer spatial vocabulary to realize improved humancomputer symbiosis. Spatial context is useful for communicating, understanding and remembering information. It includes the location of objects and people, and the place in which events are occurring. Using computers that see, we can pick out faces in a scene, track people, and follow where people are pointing and the objects they use. Using augmented reality and a real-time understanding of the spatial scene, we can overlay information on the real world or virtual models as a key spatial component of the dialog process, especially for remote virtual tele-presence, tele-supervision, and tele-science cases.
\end{abstract}

\section{Introduction}

$\mathrm{T}$ HERE is a near-term need for improved human-automation interaction in space operations. Most space systems, such as robots, docking systems, and onboard control software are controlled and monitored from ground stations. To increase flexibility and safety in the face of long-duration missions and unforeseen loss of communications, human crew must be able to monitor and control many autonomous systems at once. ${ }^{1}$ Our project is focused on providing effective team-centered communication between humans and autonomous systems via increased spatial awareness and context.

In the 1997 Mir-Progress collision in space, lack of spatial awareness was cited by Ellis ${ }^{2}$ as one of the key cognitive factors in the collision. In this docking maneuver, the radar-based Kurs automatic docking was turned off as the Progress vehicle neared MIR, and then the cosmonaut controlled the docking with the new video-based Toru

\footnotetext{
* Software Engineer, Assoc., Autonomous Systems Group, P.O. Box 179/MS 1337, AIAA Member

${ }^{\dagger}$ Systems Engineer, Sr. Staff, Autonomous Systems Group, P.O. Box 179/MS 1337, AIAA Member

$\checkmark$ Intelligent Robotics Group Lead, National Aeronautics and Space Administration, Ames Research Center, Mail Stop: 269-3, Moffett Field, CA 94035-1000, AIAA Senior Member

$\S$ Principal Investigator, Modeling, Simulation \& Information Sciences, O/ABCS B/153 C/2J5, 1111 Lockheed Martin Way, Sunnyvale, CA 94089, AIAA Member
} 
docking system. Because the automated radar docking system (Kurs) was turned off during the final approach, the cosmonaut operator had no position, range, and range rate information available. Instead, the operator only had low-contrast, narrow field of view video system to work with (Toru). Unknown to the cosmonaut, the automated Kurs system had targeted a point behind Mir instead of Mir itself, leaving the Progress vehicle with more velocity than expected. Progress collided into Mir.

Ellis points out that the crew was testing new equipment on a long duration mission, and the last time the cosmonaut operator had trained on a simulator for the complex Kurs docking system was four months prior. There was no notion of onboard docking mission-rehearsal as a refresher and test-out prior to the docking,

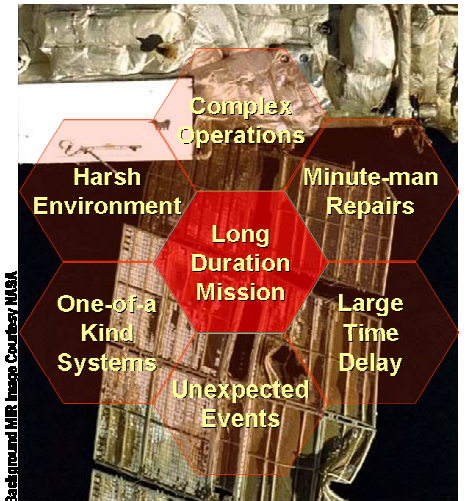

a)

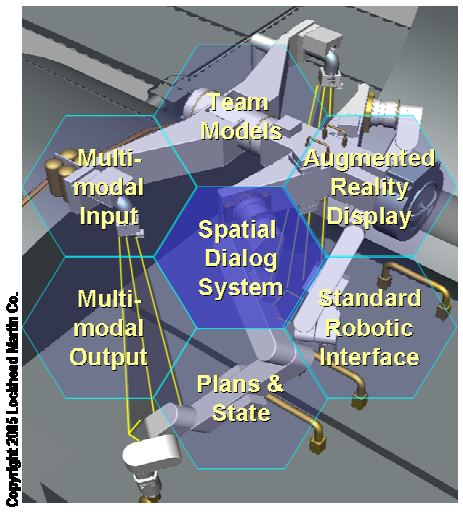

b)

Figure 1. Problems and Solutions The problems on long duration missions, such as docking collisions (a) resulting from faulty awareness of autonomy, call for better human automation interaction, and our Spatial Dialog System (SDS) provides a solution (b) with the use of spatial context for two-way team communication.

or in-situ indicators to make the settings of the Kurs autonomous docking system apparent to the operator during the docking operation.

There are a number of autonomous docking systems under development for NASA. However, the need for crew monitoring, and potential intervention during non-nominal conditions will remain. There is a similar need for all the autonomous systems on spacecraft. Crews must handle many concurrent tasks, and these require managing attention between tasks to ensure none are neglected. Errors can result from lack of spatial awareness, mode awareness, vigilance in monitoring for infrequent but important events, and faulty prospective memory for completing intended tasks.

The Mir-Progress collision example also points out a number of related problems (see Fig. 1) relevant to exploration, namely the need for training and mission rehearsal on long duration missions, the ongoing risk of trying out new one-of-a-kind systems during complex operations in a harsh environment, and when things do go unexpectedly wrong, the need to support minute-man responses and repairs such as the Mir crew had to carry out after the collision. Our solution is a Spatial Dialog System (SDS) which provides for improved team communication between humans and autonomous systems.

\section{Human Automation Interaction}

Autonomous systems are a critical element in NASA's mission for the exploration of space. However, even humans are not fully autonomous. We communicate and function with the support of others in teams, and NASA's autonomous systems, with their increasing roles, will need to communicate with humans to plan, conduct, and modify their tasks. Almost all autonomous operations will be human-centered, in response to human requests, even if they are remote. Much can be done to enable autonomous systems to communicate effectively with humans. The impact for NASA will be improved specification and understanding of autonomous system tasks for applications such as autonomous docking, in-space repair and assembly, and surface operations. The following table (Table 1) lists categories of human-automation interaction, excerpted from a recent paper on Exploration Systems CrewSystems Integration $^{3}$, which we address with our Spatial Dialog System.

With our Spatial Dialog approach, we seek to provide a common frame of reference for communication with autonomous systems by incorporating the spatial context along with the spoken context. Spatial Dialog is an interaction and communication technology that uses a deeper understanding of spatial context and a richer spatial vocabulary to realize improved human-computer symbiosis. Spatial context is useful for communicating, understanding and remembering information, and includes location and place information - the location of objects and people, and the place in which events are occurring.

Spoken dialog systems currently use speech recognition, well-defined task and discourse models, and speech synthesis, to carry on a dialog with a human on a particular topic. We hypothesize that such a dialog system can be made more robust and effective in communicating with humans when it understands more of the spatial context, and 
can use this spatial context to express itself to humans. Using computers that see, we can pick out faces in a scene, track people around a room, and follow where people are pointing and the objects they use. Using augmented reality and a real-time understanding of the spatial scene, we can overlay information on the real world as part of the dialog process.

The goal in our spatial dialog project is to improve remote virtual presence and collaboration in a data-rich environment for mixed teams of crew, autonomous systems, and remote humans. Our technical objective is to show the utility of a spatial dialog approach for improving team communication. We will concentrate on measuring error rates, completion times, and user modal preferences to show the utility of spatial dialog to accomplish mission planning, operations, and modification.

\section{A. Team-Centered Adjustable Autonomy}

We adopt a team-centered architectural approach to working with adjustably autonomous systems, which emphasizes communication between the human and robotic team members to formulate and carry out plans, monitor their execution, and modify them where necessary. According to Pell ${ }^{4}$, the issues for adjustable autonomy that must be addressed in any architectural approach include:

- Identifying opportunities for humans to interact with autonomous systems

- Enabling the human to achieve situation awareness when they have not been tracking the autonomous system

- Providing the human understanding of the implications of their actions on the overall system

- Enabling the human to safely interact with items that are too detailed for the autonomous system

Furthermore, there is often little or no mapping of system information into the proper context to provided shared situation awareness between autonomous systems and human crew members, and autonomous systems are often silent. Some adjustable autonomy issues characterized by Prinzel $^{5}$ include: $^{\circ}$

- Strong autonomy, where the system possesses complete authority and capability to carry out actions, resulting in monitoring and coordination issues for system operators

- Silent autonomy, where the system does not provide feedback on it's actions, resulting in low mode awareness for system operators

- Clumsy autonomy, where the system automates a task during low workload, and increases workload when demands are greater, resulting in an increased burden for system operators

- Complex autonomy, where the system has many interdependent controls and status data, resulting in the system operator abandoning the system for more direct approaches.

Table 1. Human-Automation Categories Addressed by Spatial Dialog System The SDS is appropriate for Mission Control, IVA \& EVA, and Training / Mission Rehearsal

\begin{tabular}{|c|c|c|}
\hline Category & Sub-Category & Relevant Spatial Dialog System Attributes \\
\hline $\begin{array}{l}\text { Mission Control } \\
\text { Operations }\end{array}$ & $\begin{array}{l}\text { Monitoring \& Control } \\
\text { Strategies }\end{array}$ & $\begin{array}{l}\text { Addresses situational awareness for vehicles, habitats, robots, by using spatial } \\
\text { attributes for explanation, planning, monitoring }\end{array}$ \\
\hline \multirow{4}{*}{$\begin{array}{l}\text { Spacecraft Systems } \\
\text { and Operations / } \\
\text { Inter-Vehicular } \\
\text { Activity }\end{array}$} & $\begin{array}{l}\text { Mission-Management } \\
\text { Systems }\end{array}$ & $\begin{array}{l}\text { Team-centered assistance for monitoring automated rendezvous and docking } \\
\text { and anomaly response and recovery. Crew use of onboard augmented reality } \\
\text { display of docking system for spatial awareness of docking procedures }\end{array}$ \\
\hline & $\begin{array}{l}\text { Human Automation } \\
\text { Interaction }\end{array}$ & $\begin{array}{l}\text { Advanced Spatial Dialog user interface supporting mixed teams of } \\
\text { crewmembers and machine based software agents. }\end{array}$ \\
\hline & $\begin{array}{l}\text { Logistics. Dynamic Real- } \\
\text { time mixed initiative }\end{array}$ & $\begin{array}{l}\text { Real-time coordination of mixed human / machine activities across team } \\
\text { members is made feasible with a spatial dialog system to create, monitor and } \\
\text { modify task plans among the team }\end{array}$ \\
\hline & Decision Support Systems & $\begin{array}{l}\text { Situation Awareness Displays can be accomplished with full spatial display of } \\
\text { relevant systems \& team members, resulting in more accurate grounding for } \\
\text { decisions }\end{array}$ \\
\hline \multirow{4}{*}{$\begin{array}{l}\text { Extra-Vehicular } \\
\text { Activity/Surface } \\
\text { Operations }\end{array}$} & $\begin{array}{l}\text { Information Displays and } \\
\text { Control }\end{array}$ & $\begin{array}{l}\text { Augmented reality with scene registration provides advanced augmented } \\
\text { reality display of data overlaid on real equipment during IVA and EVA }\end{array}$ \\
\hline & $\begin{array}{l}\text { Distributed Decision- } \\
\text { making }\end{array}$ & $\begin{array}{l}\text { Establishing the spatial context of a mission much like a hologram using } \\
\text { augmented reality and spatial dialog provides effective communication for } \\
\text { distributed decision making }\end{array}$ \\
\hline & $\begin{array}{l}\text { Human-Robotic } \\
\text { Coordination }\end{array}$ & $\begin{array}{l}\text { Providing spatial awareness and spatial reference during human and robot task } \\
\text { dialog results in faster communication and less errors in interpretation }\end{array}$ \\
\hline & $\begin{array}{l}\text { Multi-modal hands free } \\
\text { interfaces }\end{array}$ & $\begin{array}{l}\text { Spatial dialog emphasizes fused multimodal input and output for the team } \\
\text { dialog process with gaze, gesture and speech input, and 3D augmented overlays } \\
\text { and speech for robotic output }\end{array}$ \\
\hline \multirow{2}{*}{$\begin{array}{l}\text { Training and On- } \\
\text { board Decision } \\
\text { Support System }\end{array}$} & $\begin{array}{l}\text { Just-in-time Training and } \\
\text { Task Rehearsal }\end{array}$ & $\begin{array}{l}\text { IVA, EVA, and docking maneuvers can be trained against a simulation, and } \\
\text { autonomous systems plans can be reviewed and questioned }\end{array}$ \\
\hline & Team Training & $\begin{array}{l}\text { Team training in context for combined teams of humans and autonomous } \\
\text { systems via overlays on real scene, based on our team task model }\end{array}$ \\
\hline
\end{tabular}


Our approach provides an improved means of communication between adjustably autonomous systems and humans by providing a common spatial grounding for their joint tasks with a spatial model, a task model that monitors team member obligations and overall team policies, and a multimodal spatial dialog system that provides a way to interactively see and clarify tasks during the phases of their execution. These shared models are implemented in KAoS, developed by Bradshaw, et al. ${ }^{6}$

\section{B. Augmented Reality}

Augmented reality (see Fig. 2) is a display and interaction technology which overlays information on real-world scenes. Bowen et al at NASA JSC conducted a 2004 study on "Utilization of the Space Vision System As An Augmented Reality System For Mission Operations"7, and one result of their study was that appropriate augmented reality overlays offer significant improvements in robotic control

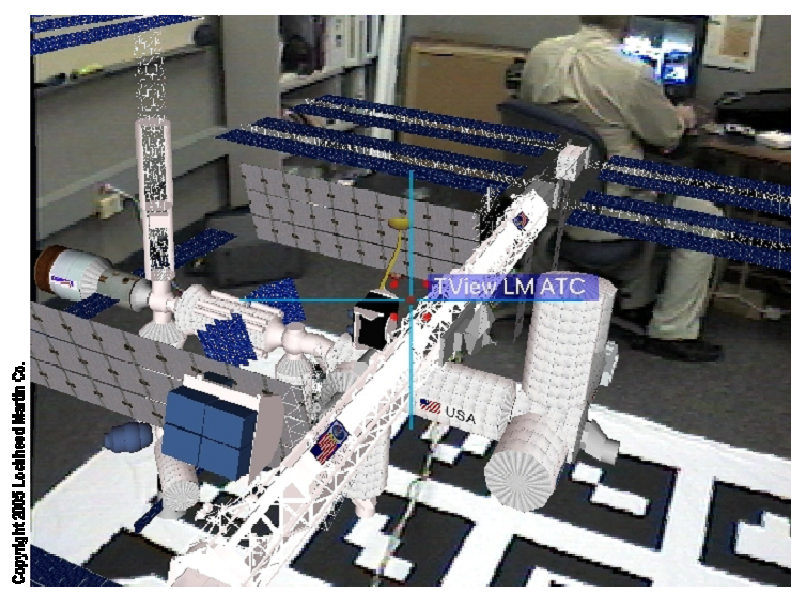

Figure 2. Space Station Augmented Reality Spatial Display This large augmented reality view of an International Space Station model enables the viewer to reach into the model to indicate items, and to move around and view the model much like a hologram. performance. Augmented reality can make plans and internal system states visible to human team members during IVA, and is even more suitable for EVA, where conventional displays and input devices are not available. Most importantly, there are more potential benefits when a robotic system can use augmented reality and speech combined to provide task explanations and status, receive task guidance, and get task clarifications from human team members ${ }^{8}$.

To create an adequate environment for communication with autonomous systems, the user must have a heightened sense of the surrounding spatial area the team member is operating in. This is known as environmental presence $^{9}$. It is necessary to move beyond simple video relay. Although video imagery provides a reasonable 2dimensional (2D) view, it is not sufficient to give a full sense of spatial awareness to the operator. To achieve effective spatial awareness it is necessary to achieve motion parallax when viewing the scene, and to effectively communicate spatial properties to others, it is necessary to use the spatial conventions such as pointing and moving objects that you would when specifying the task to a human team member.

By using augmented reality for spatial displays ${ }^{10}$, graphics can be overlaid onto real surroundings to provide a shared reference frame that is part of the dialog process. With an augmented reality display, we can overlay virtual 3D objects on physical objects for the operator, to realize a tangible user experience, where physical objects are manipulated to effect change in the shared scene. ${ }^{11}$ Thus the operator can be in a lab on earth and physically feel like they working side by side to a remote robot. The potential for team effectiveness grows quickly.

\section{Spatial Dialog}

Spatial dialog has many potential benefits by enabling humans to use spatial context to provide task guidance, clarification, and explanations to autonomous systems, as well as offering a means for autonomous systems to communicate plans and internal states to the human operator. Remote team members can highlight physical items as they are talking about them, autonomous systems can visually explain their planned actions to humans before carrying them out, and humans can visually specify or constrain the actions of autonomous systems as they work with them, enabling the team to engage in a spatial discourse about the actions the humans and autonomous systems will take.

Teams of agents (human and/or robotic) must coordinate their mental states by communication to realize a form of joint intention. The process of coordinating mental states by providing evidence is called grounding. The contribution model for the process of grounding is based on a series of contributions in the conversation, where each contribution has two phases, a presentation phase and an acceptance phase. The presentation phase is carried out to achieve the first agent's discourse goal of achieving a common understanding, and the acceptance phase, conducted by the second agent, may consist of acknowledgement, further dialog for clarification, or repair statements. Repairs are partial statements where some parts of the statement must be removed to be understood. Repair categories include removing what was said earlier to start fresh, modifications to the earlier statement, or abridged re- 
statements. We will incorporate gaze and gesture as additional repair indicators, which we call visual recovery, and could potentially increase our repair rate over that of speech alone.

In table 2, we have itemized the essential attributes of a system with spatial dialog capability. Systems must support multiple views, one for each team member, onto a shared model which includes spatial context, task information, and system state.

Table 2. Essential Spatial Dialog System Attributes This table identifies the attributes necessary for a system to have a spatial dialog capability

\begin{tabular}{|l|l|l|}
\hline Attribute & Description & \multicolumn{2}{|l|}{ Impact } \\
\hline Spatial Display & $\begin{array}{l}\text { System must display information overlaid in } \\
\text { situ with augmented reality, not on a screen or } \\
\text { PDA }\end{array}$ & $\begin{array}{l}\text { Reduce mismatch between computer \& } \\
\text { human perception. Less context switching \& } \\
\text { context loss }\end{array}$ \\
\hline Spatial Context Tracking & $\begin{array}{l}\text { System tracks user's spatial location, gaze \& } \\
\text { gestures, using 3D depth information to go } \\
\text { beyond feature-based recognition }\end{array}$ & $\begin{array}{l}\text { Better understanding for parts of dialog that } \\
\text { use spatial properties }\end{array}$ \\
\hline Spatial Dialog Model & $\begin{array}{l}\text { System must have models of how to } \\
\text { understand \& present spatial information for } \\
\text { spatial dialog, independent of task }\end{array}$ & $\begin{array}{l}\text { Reduce information overload for display, } \\
\text { better understanding when humans } \\
\text { communicate spatially, reuse across task } \\
\text { domains }\end{array}$ \\
\hline Task Model & $\begin{array}{l}\text { System must have task model which includes } \\
\text { spatial conventions to respond to task-centered } \\
\text { dialog with humans \& display of robotic plans }\end{array}$ & $\begin{array}{l}\text { Task model allows better interpretation of } \\
\text { user actions, better responses for } \\
\text { augmenting task ability \& making task } \\
\text { information visible }\end{array}$ \\
\hline Shared Information & $\begin{array}{l}\text { System components use cues from speech \& } \\
\text { spatial context tracking together }\end{array}$ & $\begin{array}{l}\text { Improved understanding of what gestures } \\
\text { mean \& what was said }\end{array}$ \\
\hline Spoken Dialog & System must have underlying spoken dialog & \begin{tabular}{l} 
System leverages spoken dialog research \\
\hline
\end{tabular}
\end{tabular}

\section{Spatial Dialog Approach}

\section{A. Operational View}

Operationally, our Spatial Dialog System is applicable to robots, vehicles, habitats, and software agents. It can be used for planning, mission rehearsal, and actual operations, which include remote docking, robotic in-space repair and assembly, and surface robotics. To illustrate our operational concept, we provide a sample day in the life using the Spatial Dialog System (SDS) for in-space repair.

It's the year 2012, and the CEV crew has encountered a problem. An unexpected failure in the Lunar Transit Vehicle's (LTV) gyroscope systems has affected the mission and they must be replaced. Because the Lunar Transit Vehicle may become unstable, the CEV has separated and is maintaining a safe distance during the repair.

Mike, the mission specialist, is communicating with Al, the autonomous system monitor, and SAM-1, a semiautonomous robot. Mike and $\mathrm{Al}$ are each using their SDS-Views to look at a shared 3D representation of the vehicle, with each mission module shown in the layout (see Fig. 3).

Mike speaks to SAM-1 and Al, "Okay SAM, let's review the module replacement plan."

SAM-1 graphically depicts his planned motion by showing a red virtual copy of his self moving along a path to the module, while at the end of robot boom, "This is the first stage, during which I open the doors where the spare modules are stored."

Sam-1 opens the doors, and moves on the robot boom to the next stage, "Next I move to the gyroscope modules on the LTV, to here."

Al points at the vehicle and asks, "Why are your cameras pointing here?"

SAM-1 replies "At this stage I must examine these mounts."

$\mathrm{Al}$ points at the most remote experiment module, "Mike, I think this module is something we can just shut down with minimal impact since it is mostly free-standing."

Mike responds by selecting two nearby modules. "Yes, but we won't power these two down until the first one is completely replaced. That will allow us one available at all times".

Mike, with a pause, asks the spatial dialog system agent to enlarge the module he is looking at, and proceeds to give instructions to SAM-1, "SAM, because these fasteners here," he says while pointing at the upper section of the 
module, "must be the first to be removed, you will have to move this blanket aside to here so you can get to the module. Before you do that, disengage these power cables."

$\mathrm{Al}$ motions to the right side of the work area, "SAM, stow the blanket here, so that our view from these cameras is not blocked", and then gestures to the left side, "and be sure to stay out of this region altogether during your operations, for the same reason."

SAM-1, who really is working with an internal task and spatial model of the LTV, responds to this request by using everyone's 3D augmented reality graphics display to show his path to the modules, "Understood, confirm that I will visit these three modules in this order," then SAM-1 shows fasteners being removed from the module, "remove these power cables, must remove and stow this blanket here, and then remove these fasteners. Where would you like the fasteners stowed?"

Al points to a storage area near the work area, and says "SAM, please put the fasteners here."

Mike, $\mathrm{Al}$ and SAM-1 work through this process for the remaining modules and SAM-1 begins his tasks. By using the spatial dialog technology, the team was able to put together a specific course of action with a minimum amount of questions, repeated sequences or false assumptions. The specification of spatial items along with speech was sufficient for them to achieve a shared model of the task at hand. In the future your robot is capable of spatial dialog; you can speak to your robot and show them the actions they should take, and they can talk with you the same way to clarify points.

With the graphical overlay of information on the team's spatial surroundings, the use of the team's surroundings for task specification, explanation, and clarification becomes much easier. Remote team members can highlight physical items when they are talking about them, autonomous systems can visually explain their planned actions to humans before carrying them out, and humans can visually specify or constrain the actions of autonomous systems as they work with them, enabling them to engage in a spatial discussion about the actions the team of humans and autonomous systems will take.

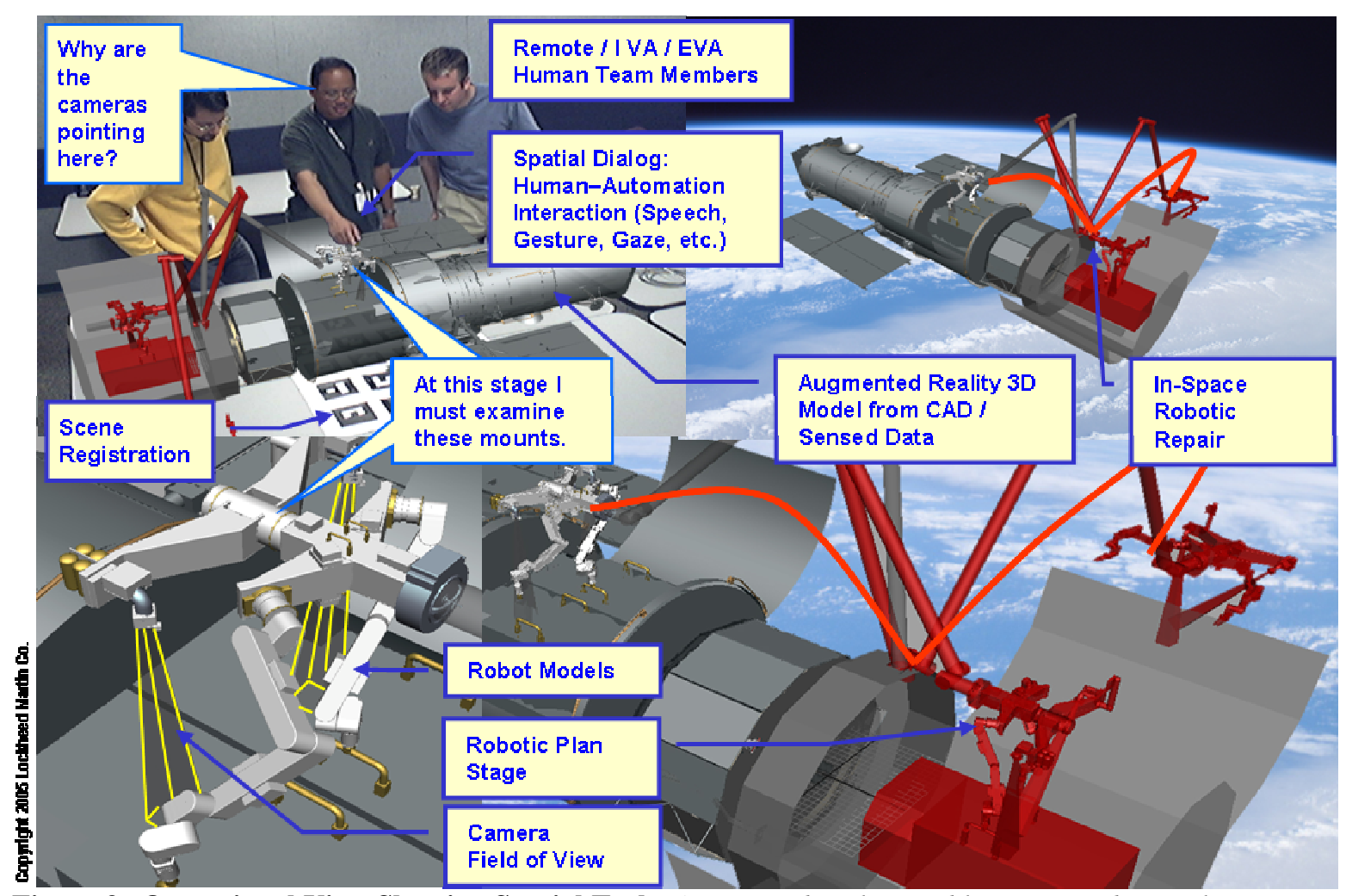

Figure 3. Operational View Showing Spatial Tasks Augmented reality enables increased spatial awareness and increased team communication by providing visual 'holographic' spatial context, where autonomous systems graphically pose questions and show their plans. 


\section{B. System View}

The Spatial Dialog System has four major components (see Fig. 4 and Table $3^{12,13}$ ); the Spatial Dialog Model, the Spatial Dialog View (often referred to as a man-machine interface or Operator Control Unit), the Spatial Dialog Control component, and the Spatial Dialog Autonomy component (sometimes referred to as a Vehicle Control Unit).

There may be one or more human team members (represented with asterisk *), each with a Spatial View onto a shared Spatial Model, and multiple autonomous clients, interacting with each other as a team. For each operating locale, there is a shared SDS-Model, where the shared spatial data, task data, and team task constraints are shared between the team members during their dialog to plan and carry out operations.

For each human team member, there is an SDS-View component and associated human interface harness which supports display and interaction with the shared spatial context. Through this harness, crew members can interact with as many autonomous systems as desired. There is an option to activate any robot or a team of robots at the same locale. It will also be possible to switch seamlessly between robots, robot teams, and computer simulations

Once a task has been developed and approved in a dialog between human team members and autonomous team members, it can be sent to the autonomous systems for execution. Lockheed Martin's Autonomous Systems Group's SDS-Autonomy client software is responsible for controlling any robotic platform in the SDS. The SDS-Autonomy component breaks down tasks through navigation and manipulation planners into lower level commands, i.e. rotate joint $B$ by 30 degrees, and reasons about behaviors necessary to execute servo controls on the robot. The SDSAutonomy client must be extensible to a variety of platforms and it must be able to guard executable plans to ensure a coordination between the actual vehicle operation and the higher level goals planned by the team.

Individual robots comprise an important category of team member in the system. They are situated in the field or in space. A robot may be a humanoid with arms or a mobile robot like the CEV itself. This robot often is autonomous and will be able to decompose high level plans to lower level plans that the robot can execute. Both components are connected by the communication system, using a set of established protocols. To make any robot or vehicle compatible with this system, it can be fitted with our "robot harness", which establishes our interfaces and software protocols. As a result, of using the robotic harness, it will be compatible with the Spatial Dialog System. Part of the robot harness is the sensor or sensors that are used to generate the SDS environment that each human team member sees in their SDS-View. These sensors include stereo vision and LIDAR.

The capabilities and command structure of every vehicle is different. There is a large difference between commanding an orbiting robot and a tracked surface vehicle. By creating a common command protocol and interpreter as a layer between the vehicle control and the rest of the system, we can produce a plug and play system for various robots to effectively and communicate the individual robot capabilities and state. This interface is responsible for updating the planners with capabilities of the robot so that only what is physically possible is incorporated into the overall strategy of the system. It is also responsible for directly commanding the autonomous system.

The SDS-Model component also includes a physics-based simulator to facilitate software development and to visualize robotic movements without physical robotic movement. The SDS-Autonomy component controls the simulation environment with the same commands that it would send to a robotic platform to support planning, mission rehearsal, and training.

To enable adjustable autonomy for members of a team, the SDS-Autonomy component incorporates a Guard. The role of the Guard is to protect the system from acting inconsistently with established policies and obligations for the team task. It compares created plans and movements prior to execution with higher level plans to ensure mission safety, efficiency, and reliability. It acts in a manner consistent with that of the KAoS policies generated by human team members during the task dialog process, thus is an added safety precaution against undesired actions that may potentially disconnect with the goals of the mission planner. 


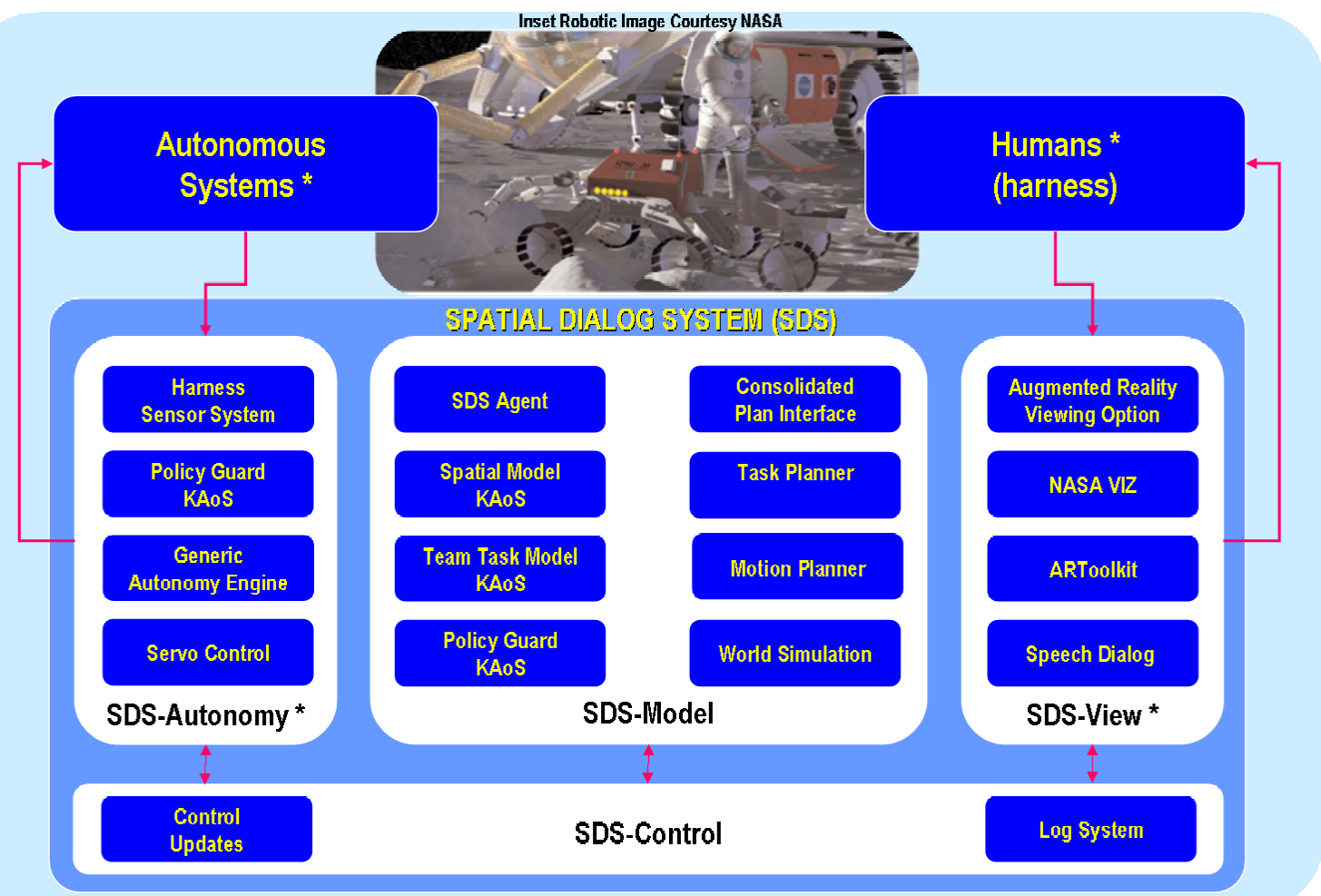

Figure 4. Spatial Dialog Component System View The Spatial Dialog System (SDS) provides two-way communication of spatial tasks for human and autonomous systems by supporting a shared spatial task model, an augmented reality view for each human, and a robotic interface for each autonomous system.

Table 3. System Component Descriptions \& Associated Hardware / Data

\begin{tabular}{|c|c|c|c|}
\hline $\begin{array}{l}\text { Com- } \\
\text { ponent }\end{array}$ & $\begin{array}{l}\text { Team } \\
\text { Member }\end{array}$ & $\begin{array}{l}\text { SDS } \\
\text { Hardware / Data }\end{array}$ & Description \\
\hline \multirow[t]{3}{*}{$\begin{array}{l}\text { SDS- } \\
\text { View }\end{array}$} & $\begin{array}{l}\text { Remote } \\
\text { Human }\end{array}$ & \multirow{2}{*}{$\begin{array}{l}\text { Human Harness; } \\
\text { Scene Tracking Cameras, Small form factor } \\
\text { Head-Mount Display, audio / microphone } \\
\text { head-set, room cameras for team location; } \\
\text { Human Spatial data, center-of-gaze, gestures, } \\
\text { speech, 3D Terrain / Scan / CAD data } \\
\text { provided or updated by SDS-Autonomy }\end{array}$} & $\begin{array}{l}\text { Provides each human's view onto the shared task area, } \\
\text { and support multi-modal input and output for spatial } \\
\text { dialog. This includes an augmented reality display and a } \\
\text { spoken dialog system for each human team member. }\end{array}$ \\
\hline & $\begin{array}{l}\text { IVA } \\
\text { Human }\end{array}$ & & $\begin{array}{l}\text { Similar to remote humans, IVA crew can control team } \\
\text { tasks from within the vehicle, interacting with external or } \\
\text { vehicle autonomous team members via the shared spatial } \\
\text { scene }\end{array}$ \\
\hline & $\begin{array}{l}\text { EVA } \\
\text { Human }\end{array}$ & $\begin{array}{l}\text { Helmet-Exterior Display, Existing Shoulder } \\
\text { Cameras, Shoulder Scanner for team location; }\end{array}$ & $\begin{array}{l}\text { For EVA, crew interact with autonomous systems using } \\
\text { an overview shared scene and first person overlays. } \\
\text { Additional scene sensing equipment is needed. }\end{array}$ \\
\hline \multirow[t]{4}{*}{$\begin{array}{l}\text { SDS- } \\
\text { Auto- } \\
\text { nomy } \\
\text { Client }\end{array}$} & Robot & $\begin{array}{l}\text { Robotic Harness - Laser Camera, Wide FOV } \\
\text { Camera; STEP CAD Files, XML Location / } \\
\text { Articulation State, Camera Locations } \\
\text { Camera FOVs, MPEG-4 Camera Feeds, } \\
\text { Periodic Laser Camera Scan Data (PIF, PLY) } \\
\text { e.g. Neptec \& Laser Dynamic Range Imager }\end{array}$ & $\begin{array}{l}\text { Provides control and sensing capabilities for each } \\
\text { autonomous team member. Some autonomy will use a } \\
\text { software-only interface; others will need additional } \\
\text { sensors for working on spatial tasks. Also connects } \\
\text { autonomous system to shared spatial model. Category } \\
\text { includes self-assembling robots, in-space repair robots, } \\
\text { and rovers. }\end{array}$ \\
\hline & Vehicle & $\begin{array}{l}\text { Data Harness } \\
\text { (subset of robotic harness) }\end{array}$ & $\begin{array}{l}\text { The vehicle category includes satellites, Crew } \\
\text { Exploration Vehicle, Modules, space stations, and } \\
\text { autonomous software / hardware systems such as health } \\
\text { systems monitoring }\end{array}$ \\
\hline & Habitat & $\begin{array}{l}\text { Data Harness } \\
\text { (subset of robotic harness) }\end{array}$ & $\begin{array}{l}\text { The habitat category includes Lunar and Martin } \\
\text { habitats, as well as associated camera systems, health } \\
\text { monitoring systems, layouts, etc. }\end{array}$ \\
\hline & $\begin{array}{l}\text { Ground } \\
\text { Control }\end{array}$ & $\begin{array}{l}\text { Data Harness } \\
\text { (subset of robotic harness) }\end{array}$ & $\begin{array}{l}\text { Some autonomy may reside with ground control systems, } \\
\text { and this component can be used here as well }\end{array}$ \\
\hline $\begin{array}{l}\text { SDS- } \\
\text { Model }\end{array}$ & Shared & $\begin{array}{l}\text { Onboard Server; Planning Domain Definition } \\
\text { Language (PDDL), Dynamic Shared Scene / } \\
\text { Motion Plans in X3D, Spatial / Team Task } \\
\text { Model in OWL }\end{array}$ & $\begin{array}{l}\text { Each local team has an assigned SDS-Model, which } \\
\text { includes the shared team task model, spatial model, } \\
\text { planning system, and world simulation. SDS-Models can } \\
\text { be connected, such as when a remote team talks to an } \\
\text { IVA team. }\end{array}$ \\
\hline
\end{tabular}

American Institute of Aeronautics and Astronautics 


\section{System Performance Metrics}

To collect formative system evaluation performance metrics for the Spatial Dialog System, we emphasize measuring human performance with the system. To this end we focus on studies to measure human team members' situation awareness and their effectiveness on critical tasks.

To set the stage for these evaluations, we are using a Wizard of $\mathrm{Oz}$ approach. In Wizard of $\mathrm{Oz}$ (WOZ) experiments, a set of subjects interacts with a software system driven by a human wizard ${ }^{14}$. The wizard operates the software, and the goal is to emulate the planned system capabilities and identify areas for improvement. Because of the complexity of multimodal interaction, we are using the WOZ studies to inform the design and implementation of our multimodal interaction system, and our subsequent system performance studies. We follow the Wizard of $\mathrm{Oz}$ with rigorous studies to assess improvements in remote situation awareness as a metric for data-rich virtual presence, and step-wise task error rate in critical procedures as a metric for communicating with adjustable autonomous systems as team members

Remote Situation Awareness - Here we use SAGAT as a metric (Situation Awareness Global Assessment Technique ${ }^{15}$ ) proven in prior studies on air traffic control and tele-operation. Below are the levels to the SAGAT measures, with the highest level of situation awareness being a predictive level. We expect higher measures of spatial situation awareness with the use of SDS than when viewed as a video.

Level 1 is perception of elements in environment

Level 2 is comprehension of current environment needed for reacting to it

Level 3 is sufficient knowledge of situation for prediction of how it will evolve

Robotic Spatial Dialog Accuracy - This metric is 1.0 - WER (Word Error Rate), the same metric used for voice recognition. We will establish a baseline SOA with speech-only dialog, and gold standard metric with a human as stand-in for autonomous system, providing an upper bound for the performance target. We expect an improvement in word error rate when compared with conventional speech recognition because additional spatial context is used.

Step-wise Error-Rate in critical procedures - This metric for error rate is the cumulative error of steps in a procedure. Critical procedures include time-critical external repair without EVA, and remote autonomous inspection/repair with a time-delay. Because of the ability to directly reference items being discussed, and greater spatial context to support discussion, we expect fewer errors to result from misunderstanding steps in procedures.

\section{Related Work}

There are a number of related efforts from the worlds of robotics, multimodal interaction and cognitive psychology that have been used to inform our approach.

On our Virtual Environments for Training (VET) project for the Office of Naval Research, we developed a team training system using virtual environments with one of the first instances of embodied conversational agents (called Soar Training Expert for Virtual Environments) that acted as mentors and missing team members for task training in the virtual environment ${ }^{16}$. The student could select objects in the world and use voice to ask the agent about the object. The student could ask for his mentor to show him a task, and then follow the mentor's gaze, hands and voice as the mentor agent showed the steps of the task. Furthermore, the student could be given a tour of the spatial layout of his task area, such as the engine room of a ship, and carry out spatial and spoken tasks with his agent teammembers. It is on this project that we first realized the significance of spatial dialog, where the emphasis is not just on fusing multimodal input, but includes the two-way exchange with multimodal output of agents situated in the world with the student.

An excellent survey of socially interactive robots, a category of robots where human-robot interaction is important, is provided by Fong et $\mathrm{al}^{17}$ In this survey, Fong notes that creating a robot that communicates at a human peer level remains a grand challenge. However, he does provide examples where task focused dialog has been achieved. Fong notes that users need explicit conversational feedback from robots before they carry out their instructions, and how some systems can use query-response dialogs as a means for humans to help robots on their tasks. Fong identifies systems that use various types of perception, such as people tracking, speech, gaze, and gesture recognition separately, but does not note any robotic systems which combine these modes for input and output, such as we propose for task-based spatial dialog.

At NASA's Jet Propulsion Laboratory, the CAMPOUT project is working to realize robotic teams that can communicate together to accomplish joint physical tasks, such as carrying beams ${ }^{18}$. This is a practical application of the robotic team advances that have appeared in the field of robotics. Our work differs in that we are talking about teams of humans and robots working together to plan, conduct, and modify tasks. 
At NASA Ames, Dorais et al. have worked to develop a Personal Satellite Assistant (PSA), a softball-sized robot that will work in teams with humans and other PSAs onboard spacecraft to support systems monitoring, communications, information updating, and remote operations ${ }^{19}$. The PSA supports a spectrum of adjustable autonomy, where it must reason about its own goals and those of other agents, including humans. To accomplish team work with humans, they have employed Brahms to model human interaction and tasks with the PSA, identified initial agent conversational policies, and have developed an initial speech interaction prototype. The PSA will operate in a complex spatial environment, and has a lot of potential for the application of spatial dialog principles; showing a crew member a particular system while telling them about it is one case. At this stage, the PSA project does not have a formal notion of spatial dialog between a human and a PSA, where a crew member gesturing or looking at an item will modify the PSA's understanding of the crew member's speech, and vice versa. Our use of augmented reality overlays for spatial output in a spatial dialog could also be of benefit for remote operation of the PSA, helping to establish a shared frame of reference, and it could be of benefit for onboard operation, allowing the PSA a means to overlay spatial information on the real scene for the crew member.

Trevor Darrel and others at the MIT AI Lab have prototyped face-pose interaction for gaze-mediated agent dialogs using stereo motion camera techniques. Using a stereo camera they set up an interactive room prototype to test different modes of interaction with agents ${ }^{20}$. They also established ideal conditions for testing these modes using a Wizard of $\mathrm{Oz}$ experiment. The modes were look-to-talk, a gaze-based approach for onset of speech, talk-to-talk, where keywords were used to determine onset of speech, and push-to-talk, a common method where a button is pressed to determine onset of speech. Although the Wizard of Oz experiment subjects said they preferred the talk-totalk method, they actually used the gaze-to-talk method to answer more of the study questions than the other modes of interaction. In our approach, based on augmented reality technology, we can identify what objects the person is looking at, effectively determining face-pose relative to these objects, and enabling a look-to-talk mode while additionally overlaying information in the spatial context as another part of the dialog process.

Teams of agents (human and/or robotic) must coordinate their mental states by communication to realize joint intention as a team. The process of coordinating mental states by providing evidence is called grounding ${ }^{21}$. The contribution model ${ }^{22}$ for the process of grounding is based on a series of contributions in the conversation, where each contribution has two phases, a presentation phase and an acceptance phase. The presentation phase is carried out to achieve the first agent's discourse goal of achieving a common understanding, and the acceptance phase, conducted by the second agent, may consist of acknowledgement, further dialog for clarification, or repair statements. Repairs are partial statements where some parts of the statement must be removed to be understood. Repair categories include removing what was said earlier to start fresh, modifications to the earlier statement, or abridged re-statements. For spoken dialog, we shall use Heeman's algorithm, based on a probabilistic assignment of words to parts of speech, for recovering from repairs ${ }^{23}$. We will be able to incorporate gaze and gesture as additional repair indicators, which we call visual recovery, and could potentially increase our repair rate over that of speech alone.

To participate in a spatial dialog, the autonomous systems must have a spatial model for changes in perspective, use of referents, interruptions, route description, and other spatial communication tasks ${ }^{24}$. The model itself must be updated based on a working solution to the correspondence problem for robotic systems, which in the robotic world is known as Simultaneous Localization and Mapping (SLAM). This is an issue not just for an embodied robots, but also for autonomous systems trying to describing how they plan to conduct an inspection, maintenance or repair task to a human team member for training, emergency operations. Our near term approach for spatial modeling is based on the use of fiducials for information on where the observer is relative to the observer. Our longer term approach is to use an absolute space representation ${ }^{25}$ where our shared model is updated relative to each observer in the team, and provide spatial updates to this model with a space-qualified 3D laser scanner capable of tracking moving objects, such as the Neptec 3D Laser Camera System used by NASA ${ }^{26}$.

\section{Conclusion}

In this paper we have described the Spatial Dialog System for Team Centered Virtual Interactive Presence with Adjustable Autonomy. Our system and approach are suitable for human-autonomous team operations, mission rehearsal, and training. Our system operates by setting a common spatial context for alerts, plans and other mission data and then supporting interactive dialog to create, monitor and modify plans of action for the mission.

Operationally, the SDS is appropriate for Remote, IVA, and EVA operations, including docking awareness and monitoring, robotic in-space repair and assembly, and surface rover operations. Within the system, we have used a model, view, control approach where humans and autonomous systems have views onto a shared spatial task model, and can discuss their plans and obligations for team tasks using spatial conventions. To this end, our system 
performance metrics are human-centered, focusing on measuring human team member situation awareness and task errors for time-critical procedures. The novel aspects of our approach include:

- Visualization: Provides augmented reality overlays as part of the formal dialog process, providing spatial context, mode awareness, and spatial awareness for remote human team members and crew

- Interaction: Addresses both input to autonomous systems and output from autonomous systems with spatial and spoken context combined, increasing the utility of each mode of interaction

- Collaboration: Enables spatial interactions across the team to provide shared awareness, grounding, and the ability to specify team tasks more directly through spatial dialog

With our Spatial Dialog System, we believe we are providing an important capability for humans to naturally collaborate with autonomous systems. It is a system that makes autonomous state, plans and action visible to improve awareness, and it supports communicating with autonomous team members in a direct manner about tasks.

\section{Acknowledgments}

We gratefully acknowledge the project contributions of our team members at NASA Ames Research Center (Larry Edwards, Dave Lees), the Florida Institute for Human Machine Computation (Jeff Bradshaw, Matt Johnson, Uszok Andrzej, Renia Jeffers), the University of Washington Human Interface Technology Lab (Tom Furness, Mark Billinghurst, Don Parker, Scott Green, Phil Lamb, Habib Abi-Rached, and Shun Ido), Omnitech Robotics (Dave Parish, Sid Gluckman, Pat Hawley), and Lockheed Martin (Peter Abeles, Bob Remington, Michael MartinezSchiferl, Catherine Harrison). We also gratefully acknowledge our project sponsor, the NASA Exploration Systems Mission Directorate Software \& Intelligent Systems \& Modeling Program Element under contract NNA05BE41C, administered by the NASA Ames Research Center. The opinions expressed in this paper are our own and not necessarily those of our sponsor.

\section{References}

${ }^{1}$ Korsmeyer, D. Clancy, D. Crawford, J. Drummond, M., "Crew Centered Operations: What HAL 9000 Should Have Been", AIAA-2005-2546, Proc. 1st Space Exploration Conference, Orlando, Florida, Jan. 30-1, 2005.

${ }^{2}$ Ellis, S., "Collision in Space", Ergonomics in Design, Winter, 2000.

${ }^{3}$ Allard, T., "The New Explorers: ESMD Crew-Systems Integration Research \& Vision", AIAA-2005-2546, Proc. 1st Space Exploration Conference, Orlando, Florida, Jan. 30-1, 2005.

${ }^{4}$ Pell, B., Shafto, M., "Adjustable Autonomy in NASA's Exploration Vision", Proc. of AIAA $1^{\text {st }}$ Intelligent Systems Technical Conference, Chicago, IL, AIAA 2004-6370, September, 2004.

${ }^{5}$ Prinzel, L. J., "Team-Centered Perspective for Adaptive Automation Design”, NASA TM-2003-212154, NASA Langley

Research Center, Hampton, VA, 2003.

${ }^{6}$ Bradshaw, J., Dutfield, S., Carpenter, B., Jeffers. R., Robinson, T., "KAoS: A Generic Agent Architecture for Aerospace Applications", Proc. CIKM '95 Workshop on Intelligent Information Agents, Baltimore, MD, 1995.

${ }^{7}$ Bowen, C., Maida, J., Montpool, A., Pace, J., "Utilization of the Space Vision System As An Augmented Reality System For Mission Operations", Proc. AIAA Habitation Conference, HFE28, Houston, TX, 2004.

${ }^{8}$ Billinghurst, M., Weghorst, S., Furness, T., "Wearable Computers for Three Dimensional CSCW", Proc. International Symposium on Wearable Computers, Cambridge, MA, October 13-14, 1997. Los Alamitos, IEEE Press, pp. 39-46.

${ }^{9}$ Heeter, C. "Being There: The subjective experience of presence," Presence: Teleoperators and Virtual Environments, MIT Press, fall, 1992.

${ }^{10}$ Chinthammit,W., Seibel, E., Furness, T., “A Shared-Aperture Tracking Display for Augmented Reality”, Presence: Teleoperators and Virtual Environments, 12(1), 1-18, 2003.

${ }^{11}$ Billinghurst, M., Grasset, R., Looser, J., "Designing Augmented Reality Interfaces", Computer Graphics SIGGRAPH Quarterly, 39(1), February, 2005, p17-22

${ }^{12}$ For XML-based formats, see http://www.w3.org/Consortium/siteindex.html

${ }^{13}$ For Scanned Point Cloud formats, see http://www.innovmetric.com

${ }^{14}$ Bernsen, N. O., Dybkjær, H., and Dybkjær, L., Designing Interactive Speech Systems - From First Ideas to User Testing. Springer. 1998.

${ }^{15}$ Endsley, M. R., Garland D. J. (eds.) Direct Measurement of Situation Awareness Validity and use of SAGAT. In Situation Awareness Analysis and Measurement, Mahwah, NJ Lawrence Erlbaum Assoaciates, 2000.

16 Johnson, W. L., Rickel, J., Stiles, R., and Munro, A., "Integrating Pedagogical Agents into Virtual Environments". Presence Journal 7(6), MIT Press, Dec 1998.

${ }^{17}$ Fong, T., Nourbakhsh, I., Dutenhahn, K., "A Survey of Socially Interactive Robots: Concepts, Design, and Applications", Carnegie Mellon University Technical Report CMU-RI-TR-02-29, 2002. 
${ }^{18}$ Huntsberger, T., Pirjanian, P., Trebi-Ollennu, A., Nayar, H. D., Aghazarian, H., Ganino, A., Garrett, M., Joshi, S., Schenker, P. S., "CAMPOUT: A Control Architecture for Tightly Coupled Coordination of Multi-Robot Systems for Planetary Surface Exploration," IEEE Trans. Systems, Man \& Cybernetics, Special Issue on Collective Intelligence, 2003.

${ }^{19}$ Dorais, G., Nicewarner, K., "Adjustably Autonomous Multi-agent Plan Execution with an Internal Spacecraft Free-Flying Robot Prototype", Proc. ICAPS 03, Trento, Italy, June, 2003

${ }^{20}$ Darrell, T., Tollmar, K., Bentley, F., Checka, N., Morency, L., Rahimi, A., Oh, A., Face-responsive interfaces: from direct manipulation to perceptive presence, International Conference of Ubiquitous Computing, 2002.

${ }^{21}$ Clark, H. H., and Brennan, S. E., Grounding in Communication. In L.B. Resnick, J. Levine, and S.D. Teasley, eds., Perspectives on Socially Shared Cognition, 127-149. Washington DC: APA. 1991.

${ }^{22}$ Clark, H. H., and Schaefer, E. F., "Contributing to Discourse”, Cognitive Science, 13:259-294. 1989.

${ }^{23}$ Heeman, P., and Allen, J., Speech Repairs, 'Intonational Phrases and Discourse Markers: Modeling Speakers' Utterances in Spoken Dialog", Computational Linguistics, Vol. 25-4, 1999.

${ }^{24}$ Bateman, J. Farrar, S., "Modeling Models of Robot Navigation Using Formal Spatial Ontology", in Proc. Intl. Conf. Spatial Cognition, Frauenchiemsee, Germany, October, 2004. pp. 366-389.

${ }^{25}$ Jeffries, M., Cree, M., May, M., Baker, J., "Using 2D and 3D Landmarks to Solve the Correspondence Problem in Cognitive Robot Mapping”, in Proc. Intl. Conf. Spatial Cognition, Frauenchiemsee, Germany, October, 2004, pp. $434-454$.

${ }^{26}$ Samson, C., English, C., Deslauriers, A., Christie, I., Blais, F., Ferrie, F. "Neptec 3D Laser Camera System: From Space Mission STS-105 to Terrestrial Application”, Canadian Aeronautics and Space Journal, NRC 46565, June 2004. 